\title{
3 Research Square

\section{Association of Oral Microbiota with Sugar-Sweetened Beverages Consumption and Oral Health Problems in School-Aged Children}

\section{Xin Chen}

Anhui Medical University

Xiaoyan Hu

Anhui Medical University

Jiao Fang ( $\sim$ fangjiao@stu.ahmu.edu.cn )

Anhui Medical University https://orcid.org/0000-0002-6508-1433

Xiaoyu Sun

Anhui Medical University

Fangfang Zhu

Anhui Medical University

Ying Sun

Anhui Medical University

Yuanyin Wang ( $\nabla$ wangyuanyin@ahmu.edu.cn )

Anhui Medical University

\section{Research article}

Keywords: Sugar-sweetened beverages, Oral microbiota, Oral health problems

Posted Date: May 13th, 2020

DOl: https://doi.org/10.21203/rs.3.rs-28097/v1

License: (c) (1) This work is licensed under a Creative Commons Attribution 4.0 International License. Read Full License 


\section{Abstract}

Consumption of beverages is prevalent among children and adolescents, of which the most popular beverages were sugar-sweetened beverages (SSBs). There is evidence that common beverage consumption, like coffee and tea, is associated with oral ecosystem. However, little is known about the effect of SSBs on the composition and functional potential of childhood oral microbiota.To examine the associations between SSBs consumption with oral microbiota diversity among school-aged children.Data came from the fourth wave follow-up of an ongoing child growth and development cohort established in 2016. Oral microbiota in buccal cheek swab samples were collected from 180 children (78 boys and 112 girls, aged 11.33 \pm 0.55 years old) using $16 S$ rDNA gene sequencing. Students' questionnaire was used to collect SSB consumption. Oral health problems, such as dental caries, gingivitis, was examined by a professional dentist. Higher SSBs consumption ( $\geq 1$ serving/week) was associated with lower oral microbiota richness and diversity. Children with higher SSBs consumption showed altered abundance of several oral microbiota at genus level, including increases in Streptococcus, Gemella, Neisseria,Capnocytophaga and Lautropia (and decreases in Fusobacterium, Lachnoanaerobaculum, Soonwooa, Tannerella and Moraxella $(p<0.05)$. Functional analysis illustrated that oral microbiota was more conducive to the pathway activated of lysine biosynthesis $(p=0.045)$, protein export $(p=0.020)$, D-glutamine and $\mathrm{D}$-glutamate metabolism $(p=0.013)$, as well as pantothenate and CoA biosynthesis $(p=0.004)$ in children with higher SSBs intake. Our finding suggests that higher SSBs consumption may disturb oral microecology and reduce diversity and abundance of oral microbiota during childhood.

\section{Introduction}

The oral cavity is among the most heavily colonized areas of the human body and harbors the second most diverse human microbiome ${ }^{[1]}$. Previous studies of the oral microbiome have estimated the presence of up to 700 bacterial species ${ }^{[2]}$, plays a central role in the maintenance of oral health ${ }^{[3]}$, of which approximately one third cannot be cultured ${ }^{[4-6]}$. The mouth is also the site where the most prevalent human diseases occur, including caries, gingivitis, and periodontal disease ${ }^{[1.7,8]}$, and oral dysbiosis has been associated with systemic diseases, such as diabetes, cardiovascular disease ${ }^{[1,6]}$ and cancers (head and neck, pancreatic and esophageal cancer) ${ }^{[9-}$ 11]. While the importance of the oral microbiome in human health is becoming increasingly clear, little is known regarding factors that influence oral microbiome composition. Previous studies suggest that long-term dietary habits have been shown to affect the diversity and composition of the human gut microbial community ${ }^{[12-14]}$. Few studies have explored the contribution of beverages consumption e.g. sugar-sweetened beverages in shaping the oral microbial community.

Over-consumption of dietary sugar is a public health concern ${ }^{[15]}$, especially in children and adolescents. A study of 2032 children aged from 6 to 18 reported that $21.7 \%$ Chinese children had more than one SSB serving per week, while $9.5 \%$ had one or more SSB servings per day ${ }^{[16]}$. The national nutrition survey in Australia indicated that daily intake of added sugars is highest (exceeds recommended intake) among adolescents ${ }^{[17]}$, and SSBs are the main food sources of added sugars ${ }^{[18,19]}$. Intake of added sugars, particularly sugar sweetened beverages (SSBs) may reduce the intake of more nutritious foods, leading to a poor quality diet, contributing to the development of dental caries ${ }^{[20,21]}$ that is associated with shifts of microbiota in dental biofilms ${ }^{[22]}$. In addition, studies have demonstrated that frequent intake of sugars (especially sucrose) and other carbohydrates lowers the $\mathrm{pH}$ and changes the microenvironment of the mouth, influencing plaque bacteria ${ }^{[20,22-25]}$. Other 
evidence suggests that excessive consumption of acidic drinks or refined sugar could further impact on the oral

ecosystem ${ }^{[26-28]}$, which could be a further mechanism for the effects of these beverages on oral and/or systemic health, including their chemopreventive properties. However, previous studies have focused on adults, or very young infants, with studies on children and adolescents lagging behind, and that the associations of sugarsweetened beverages with oral microbiota diversity and composition have not been well investigated.

The current study collected buccal swabs samples from the buccal mucosa of 180 school-aged children from an ongoing child growth and development cohort in China (78 boys, 102 girls, ranging in age from 10.33 to 12.50 years). Oral microbiota was assessed via next-generation $16 \mathrm{~S}$ rRNA gene sequencing ${ }^{[29,30]}$, and oral microbiome diversity and composition were evaluated in relation to frequency of coffee and tea intake.

\section{Methods}

\section{Study Population}

The present study used data from the fourth wave follow-up of an established child growth and development cohort longitudinal study, which recruited grade 1 to 3 from 2 primary school of Bengbu city, Anhui province in March 2016. Written informed consent was obtained from parents of all children. Questionnaires were used to collect demographics and sugar-sweetened beverages (SSBs) consumption information annually. Buccal swab samples were collected by rubbing the inside of both cheeks with a swab at the last follow-up. And a random sample of 202 children were selected for oral microbiota detection by using 16S rDNA sequencing. The final sample of who had available beverage consumption and oral microbiota data consisted of 180 children (78 boys, 102 girls). Average age was $11.33 \pm 0.55$ (range from 10.33 to 12.50) years and mean BMI was $19.55 \pm$ $3.83(13.57-31.93) \mathrm{kg} / \mathrm{m}^{2}$. Ethics approval of this study was granted by the Institutional Review Boards at Anhui Medical University (No. 20160112),

\section{Measurements}

\section{General information}

Birth and family-related information was collected through parent questionnaire at baseline survey, including birth weight, early feeding mode (exclusive breastfeeding, formula milk and mixed feeding), delivery mode (natural delivery and cesarean section), gestational age, monthly household income, parental education level.

\section{Sugar sweetened beverages consumption}

Sugar sweetened beverages (SSBs) consumption was measured with the question from children: "During the past week, how many times have you drunk sugar-sweetened beverages (juice drinks, carbonated drinks, milk tea, tea drinks, etc.) ?" with responses alternatives: 0 serving/week (no intake), $<1$ serving/week, $\geq 1$ and $<3$ servings/week, and $\geq 3$ servings/week. In this data analysis, participants were categorized into 2 categories as follows: $<1$ serving/week (low intake group, $n=150$ ) and $\geq 1$ servings/week (higher intake group, $n=30$ )

\section{Oral health examination}




\section{Buccal swabs collection and 16S rDNA sequencing}

Buccal swabs were collected by trained researcher according to a standardized protocol. No eating and drinking 30 minutes before sampling, buccal swab samples were collected by rubbing the inside of both cheeks with a swab for 10 to 15 times. Break off one end with a cotton brush and soak it in the preservation solution, ensured that sample is thoroughly soaked. All specimens were stored at $-20^{\circ} \mathrm{C}$ and waited for processing in the lab.

Total bacterial DNA was extracted by PowerSoil ${ }^{\text {TM }}$ DNA Isolation Kit (MoBio, CA, USA) and operated according to the Kit instructions. DNA quality was evaluated on agarose gel after extraction, and the amount of DNA was determined by Nanodrop 2000 (Thermo Fisher Scientific, USA) and Invitrogen Qubit 3.0 spectrophotometer (Thermo Fisher Scientific, USA). The V3 + V4 region of the 16S rRNA gene was amplified by PCR using universal primers 805 R (5'-ACTACHVGGGTATCTAATCC-3') and 341 F (5'-CCTACGGGNGGCWGCAG-3'). The PCR products were purified and sequenced using Illumina MiSeq/HiSeq platform. PCR efficiencies were obtained between 98 and $106 \%$, with $R^{2}$ values $>0.99$. The quality sequences were then clustered into operational taxonomic units (OTU) at the 0.03 level; the most abundant sequence was chosen as a representative sequence for each OTU.

\section{Body mass index}

Annual physical examination was measured in duplicate during school hours. Height and weight were measured with an integrated height and weight measuring instrument, and BMI was calculated as weight (in kg) divided by height squared (in $\mathrm{m}$ ).

\section{Statistical analysis}

We compare the differences in age, BMI, family economic status, birth information and the detection rate of oral problems among different groups of SSBs consumption using independent sample t test, chi-square test and Fisher's exact probability method. Spearman rank correlation was used for correlation analysis of the relative abundance of the flora with age and oral health problems. Statistical significance was accepted at $p<0.05$. SPSS 23.0 was used for general statistics analysis.

Original data obtained by bioinformatics analysis and sequencing were filtered out of low-quality reads. The OTUs were aligned to the Ribosomal Database Project (RDP) database using mothur (classify.seqs) for the annotation of genus and species ${ }^{[31]}$. Non-metric multidimensional scaling (nMDS) analysis was applied to investigate the genus differences between the two groups using the "vegan" package in R (version 3.6.2) ${ }^{[32]}$. The taxonomic alpha diversity (as OTU, Chao1 and Shannon index) indices was calculated using the QIIME software. Metastats analysis was profiled to identify difference of oral bacterial genera among children with different SSBs intake. Oral microbiota functions were predicted on the basis of 16S rDNA OTUs profiling using PICRUSt with default parameters ${ }^{[33]}$.

\section{Results}


In this study population, $16.7 \%(30 / 180)$ of children reported higher sugar-sweetened beverages (SSBs) intake ( $\geq$ 1 serving/week). Those with higher SSBs intake were more likely to be boys. Table 1 presents description of demographic variables and oral health problems based on frequency of SSBs consumption. Compared with lower intake group, prevalence of dental caries $\left(40.00 \%\right.$ vs. $\left.21.33 \%, \chi^{2}=4.717, p=0.030\right)$ and gingivitis $(20.00 \%$ vs. $\left.3.33 \%, \chi^{2}=12.103, p=0.001\right)$ was significantly higher among higher SSBs intake group.

Table 1

Demographic, oral health problems by different SSBs frequency groups

\begin{tabular}{|c|c|c|c|c|}
\hline & \multicolumn{2}{|c|}{ Sugar-sweetened beverages (SSBs) consumption groups } & \multirow{2}{*}{$x^{2} / t$} & \multirow[t]{2}{*}{$p$-value } \\
\hline & $<1$ serving/week $(n=150)$ & $\geq 1$ serving/week $(n=30)$ & & \\
\hline Girls (\%) & $90(60.00)$ & $12(40.00)$ & 4.072 & 0.044 \\
\hline $\mathrm{BMI}\left(\mathrm{kg} / \mathrm{m}^{2}\right)$ & $19.39 \pm 3.62$ & $20.37 \pm 4.73$ & 1.072 & 0.202 \\
\hline BMI status & & & 1.199 & 0.549 \\
\hline normal & $92(61.33)$ & $20(66.67)$ & & \\
\hline overweight & $29(19.33)$ & $4(13.33)$ & & \\
\hline obesity & $29(19.33)$ & $6(20.00)$ & & \\
\hline Dental caries & & & 4.717 & 0.030 \\
\hline No & $118(78.67)$ & $18(60.00)$ & & \\
\hline Yes & $32(21.33)$ & $12(40.00)$ & & \\
\hline Gingivitis & & & 12.103 & 0.001 \\
\hline No & $145(96.67)$ & $24(80.00)$ & & \\
\hline Yes & $5(3.33)$ & $6(20.00)$ & & \\
\hline Debris index & & & 0.755 & 0.792 \\
\hline No & $81(54.00)$ & $13(43.33)$ & & \\
\hline Tip edge & 47 (31.33) & $12(40.00)$ & & \\
\hline$\geq 1 / 3$ coverage & $22(14.67)$ & $5(16.67)$ & & \\
\hline
\end{tabular}

The library coverage of 180 sequencing samples exceeded $98 \%$, and 27853 OTUs was obtained through sequence clustering. Compare to those who consumed SSBs less than one serving per week, children with $\geq 1$ serving/week intake of SSBs showed lower species richness community diversity as reflected by a smaller Chao 1, abundance-based coverage estimators (ACE), Shannon index, as well as a higher Simpson index (Table 2). 
Table 2

Comparisons of a diversity indices among children with different sugar-sweetened beverages preferences

$\begin{array}{lllll}\text { Groups } & n & \text { Chao }^{a} & \text { ACE } & \end{array}$ Shannon index Simpson's index

\section{Sugar-sweetened beverages (SSBs)}

$\begin{array}{lccccc}<1 \text { serving/week } & 150 & 230.34 \pm 52.60 & 230.88 \pm 47.14 & 2.90 \pm 0.49 & 0.13 \pm 0.07 \\ \geq 1 \text { serving/week } & 30 & 216.39 \pm 55.01 & 212.69 \pm 47.14 & 2.82 \pm 0.52 & 0.14 \pm 0.08\end{array}$

a Abundance-based estimator of species richness; ${ }^{b}$ Abundance-based coverage estimator of species richness

A total of 260 genera was detected at the genus level, and Fig. 1 and Supplementary Table 1 shows 17 genera with relatively high abundance in two SSBs consumption groups. Compared to the lower SSBs intake group (< 1 serving/week), children with higher SSBs intake ( $\geq 1$ serving/week) showed a higher relative abundance of oral microbiota included Neisseria (12.73\% vs. $11.20 \%)$, Gemella (6.37\% vs. 5.93\%), Porphyromonas (3.32\% vs. $2.93 \%$ ), Actinomyces (2.58\% vs. 3.10\%), Lautropia (3.02\% vs. $2.13 \%)$, Granulicatella (2.23\% vs. $1.90 \%)$, Fusobacterium (2.29\% vs. $1.77 \%)$, Alloprevotella (1.54\% vs. $1.43 \%$ ) and Capnocytophaga (1.68\% vs. $1.33 \%)$; and a lower relative abundance of Streptococcus (28.00\% vs. 28.27), Gemella (6.17\% vs. 6.27), Haemophilus (12.17\% vs. 12.40$)$, Veillonella (3.98\% vs. 5.07), Leptotrichia (3.68\% vs. 3.87), Rothia (3.02\% vs. 4.30$)$, Prevotella (2.16\% vs. 2.43), Corynebacterium (1.41\% vs. 2.10) and Aggregatibacter(0.95\% vs. 1.13).

At phylum level, the dominant microbiota included high relative abundance of Actinobacteria among children with higher SSBs intake, and high relative abundance of Firmicutes, Proteobacteria, Bacteroidetes, Fusobacteria, Candidatus_Saccharibacteria, SR1, Spirochaetes and Cyanobacteria/Chloroplast in children with lower SSBs intake (Fig. 1 and Supplementary Table 2).

Higher SSBs intake was associated with greater abundance of several oral microbiota at genus level, including Streptococcus and Gemella (phylum Firmicutes), Neisseria, Capnocytophaga (phylum Bacteroidetes) and Lautropia (phylum Proteobacteria) (Fig. 2 and Supplementary Table 3). It was also related to lower abundance of genus Fusobacterium (phylum Fusobacteria), Lachnoanaerobaculum (phylum Firmicutes), Soonwooa and Tannerella (phylum Bacteroidetes), Moraxella (phylum Proteobacteria).

For children with higher SSBs intake, oral microbiota was more conducive to the pathway activated of lysine biosynthesis $(p=0.045)$, protein export $(p=0.020)$, D-glutamine and D-glutamate metabolism $(p=0.013)$ and pantothenate and CoA biosynthesis ( $p=0.004)$ (Fig. 3 and Supplementary Table 4). As illustrated in Table 3, according to the results of different SSBs consumption groups, the incidence of dental caries in children who consumed higher than 1 serving/week of SSBs is related to the increase in the relative abundance of Streptococcus ( $r=0.246, p=0.007)$ and Capnocytophaga $(r=0.206, p=0.041)$, and the decrease in the relative abundance of Fusobacterium ( $r=-0.194, p=0.018)$. Children who consumed less than one serving per week of SSBs showed a decrease in Lachnoanaerobaculum $(r=-0.173, p=0.034)$. The incidence of gingivitis in children with SSBs intake $\geq 1$ serving/week is related to the decrease in the relative abundance of Lachnoanaerobaculum $(r=-0.184, p=0.037)$. 
Table 3

Correlation of different oral microbiota and oral health problems at genus level

\begin{tabular}{|c|c|c|c|c|c|c|}
\hline & \multicolumn{3}{|c|}{$\begin{array}{l}\text { SSBs intake }<1 \text { serving/week }(n= \\
150)\end{array}$} & \multicolumn{3}{|c|}{$\begin{array}{l}\text { SSBs intake } \geq 1 \text { serving/week }(n= \\
\text { 30) }\end{array}$} \\
\hline & Dental caries & Gingivitis & $\begin{array}{l}\text { Debris } \\
\text { Index }\end{array}$ & Dental caries & Gingivitis & $\begin{array}{l}\text { Debris } \\
\text { Index }\end{array}$ \\
\hline Streptococcus & $0.163^{*}$ & 0.054 & -0.004 & $0.246 * *$ & 0.064 & -0.145 \\
\hline Neisseria & -0.076 & -0.115 & 0.032 & 0.117 & 0.102 & 0.218 \\
\hline Gemella & -0.044 & 0.010 & -0.060 & -0.301 & 0.076 & 0.051 \\
\hline Lautropia & -0.042 & 0.087 & 0.028 & 0.187 & -0.045 & 0.096 \\
\hline Capnocytophaga & 0.130 & -0.109 & 0.137 & $0.206^{*}$ & -0.007 & 0.309 \\
\hline Soonwooa & -0.137 & 0.126 & -0.029 & 0.351 & 0.113 & 0.181 \\
\hline Moraxella & 0.093 & -0.120 & 0.031 & 0.083 & -0.091 & 0.315 \\
\hline Lachnoanaerobaculum & $-0.173^{*}$ & 0.092 & 0.070 & 0.201 & $-0.184^{*}$ & 0.137 \\
\hline Fusobacterium & -0.122 & -0.011 & 0.036 & $-0.194^{*}$ & -0.137 & 0.037 \\
\hline Tannerella & 0.154 & -0.122 & 0.127 & 0.213 & -0.168 & 0.168 \\
\hline${ }^{\star} p<0.05,{ }^{* \star} p$ & & & & & & \\
\hline
\end{tabular}

\section{Discussion}

The findings of the present study showed, for the first time, that sugar sweetened beverages (SSBs) intake significantly altered the diversity and composition of the oral microbiota among children. More specifically, compared with lower intake group, higher consumption of SSBs ( $\geq 1$ serving/week) was associated with lower oral microbiota diversity, and altered abundance of several genera, including lower abundance of Fusobacterium, Lachnoanaerobaculum, Soonwooa, Tannerella and Moraxella, and higher abundance of Streptococcus, Gemella, Neisseria, Capnocytophaga and Lautropia.

The ecological plaque hypothesis ${ }^{[34,35]}$ proposed that environmental changes in oral conditions, such as diet habits ${ }^{[36]}$, were responsible for a population balance shift of the resident microbiota. Keller et al. compared the oral microbial profiles of 90 young adults with different levels of sugar intake, and found that participants of the low-sugar group were less frequently colonized by $S$. sobrinus and P. melaninogenica and displayed lower levels of a number of caries-associated bacteria such as S. mutans, S. wiggsiae, Veillonella, and Actinomyces species [37]. Tian et al. collected supragingival plaque from 24 children aged $2-4$ years who underwent one caries treatment session, finding a positive correlation between sugar intake frequencies and several bacteria species in co-occurrence networks, including Selenomonas, Treponema, Prevotella, Capnocytophaga, and Mitsuokella, which could metabolize sugar and generate acid ${ }^{[38]}$. Though the association between sugar consumption with altered the salivary microbial profiles has been shown previously among adults and younger children, less is known about the impact of SSBs intake on the composition and function of the oral microbiota, especially among school-aged children. The present study seeks to address this issue illustrating the relative abundance of 
Streptococcus, Gemella, Neisseria, Capnocytophaga and Lautropia increased in children with SSBs intake higher than one serving per week, and decreased relative abundance of Fusobacterium, Lachnoanaerobaculum, Soonwooa, Tannerella and Moraxella.

Previous studies revealed that community structure shifts of oral microbiota are more closely associated with childhood cariogenesis ${ }^{[39-42]}$. Clinical studies have linked Streptococcus mutans and Streptococcus sobrinus to both caries progression ${ }^{[43]}$ and early childhood caries ${ }^{[44]}$, and the concurrent appearance of those microbiota seem to be strongly associated with caries status ${ }^{[45.46]}$.

Some studies have found that the numbers of Streptococcus mutans, Lactobacillus sp., and some acidic bacteria decrease after caries therapy, especially in relapse-free children ${ }^{[44,47]}$. Al-Ahmad et al. ${ }^{[48]}$ evaluated changes in 13 children's oral health and oral biofilm composition in vivo during poor diet and oral hygiene, indicating that the decreased proportion of Fusobacterium nucleatum and increased proportion of Veillonella spp. across adults with dental caries. Early molecular methods studies have shown that putative periodontal pathogens such as Prevotella gingivalis and Actinomyces actinomycetemcomitans have been identified to be associated with saliva samples from adult patients with periodontitis ${ }^{[49]}$ and Streptococcus mutans with samples from carious lesions of children aged 10 to 14 years ${ }^{[50]}$. The current findings are consistent with the above conclusions, showing a significant positive correlation between Streptococcus and dental caries in children and negative relationship of Fusobacterium and dental caries. In addition, we found that another oral microbiota (Capnocytophaga) related to low prevalence of dental caries, and Lachnoanaerobaculum was negatively associated with prevalence of gingivitis.

There are some weaknesses in the interpretation of our findings. Firstly, data of SSBs consumption was selfreported by children and thus susceptible to report bias due to socially desirable responses. Secondly, the crosssectional analysis limits causal inferences allowing us to report only associations. In addition, we only reported the effect of SSBs on oral microbiota, which may be influenced by sugary diet or other beverages. Further study might offer insights into the bi-directional impact between beverages intake (e.g. SSBs) or diet habits and oral microbiota composition. Finally, we only identified the difference of oral microbiota by using 16S rDNA, the promising oral microbiota metabolomics approaches should be further considered in the future. Despite these limitations, there were some strengths including the oral health questions was derived from dental examinations by two professional dentists, ensuring that the data is authentic and reliable.

\section{Conclusion}

In summary, our findings suggest higher SSBs consumption may decrease diversity and alter overall composition of the oral microbiota community during childhood. These findings may have implications for better understanding the potential role that oral bacteria play in SSBs-related oral diseases among children.

\section{Declarations}

\section{Ethics approval and consent to participate:}

Not applicable. 


\section{Consent for publication:}

Not applicable.

\section{Availability of data and materials:}

The datasets used and/or analysed during the current study are available from the corresponding author on reasonable request.

\section{Competing interests:}

The authors declare that they have no competing interests.

\section{Funding:}

This work was supported by National Natural Science Foundation of China (grant number 81872638) and Key Project of Education Bureau of Anhui Province (KJ2019A0252).

\section{Authors' contributions:}

SY and WYY conceptualized and designed this study. Acquisition of data: CX, FJ, HXY, SXY, ZFF and SY analyzed and interpreted the data. CX, SY and WYY revised manuscript for intellectual content. All authors read and approved the final manuscript.

\section{Acknowledgements:}

Not applicable

\section{References}

1. Kilian M, Chapple ILC, Hannig M, Marsh PD, Meuric V, Pedersen AM, et al. The oral microbiome-an update for oral healthcare professionals. Bdj. 2016;221:657-66.

2. Dewhirst FE, Chen T, Izard J, Paster BJ, Tanner AC, Yu WH, et al. The human oral microbiome. J bacteriol. 2010;192(19):5002-17.

3. Wade WG. The oral microbiome in health and disease. Pharmacol Res. 2013;69(1):137-43.

4. Chen $\mathrm{H}$, Jiang W. Application of high-throughput sequencing in understanding human oral microbiome related with health and disease. Front Microbiol. 2014;5:508.

5. Hajishengallis E, Parsaei Y, Klein MI, Koo H. Advances in the microbial etiology and pathogenesis of early childhood caries. Mol Oral Microbiol. 2017;32:24-34.

6. He J, Li Y, Cao Y, Xue J, Zhou X. The oral microbiome diversity and its relation to human diseases. Folia Microbiol (Praha). 2015;60:69-80. 
7. Belstrøm D, Constancias F, Liu Y, Yang L, Drautz-Moses DI, Schuster SC, et al. Metagenomic and metatranscriptomic analysis of saliva reveals disease associated microbiota in patients with periodontitis and dental caries. NPJ Biofilms Microbiomes. 2017;3:23.

8. Kageyama S, Takeshita T, Asakawa M, Shibata Y, Takeuchi K, Yamanaka W, et al. Relative abundance of total subgingival plaque-specific bacteria in salivary microbiota reflects the overall periodontal condition in patients with periodontitis. PLoS One. 2017;12:e0174782.

9. Hayes RB, Ahn J, Fan X, Peters BA, Ma Y, Yang L, et al. A Prospective Study of Oral Microbiome and Risk for Incident Head and Neck Squamous Cell Cancer. JAMA Oncol. 2017.

10. Fan X, Alekseyenko AV, Wu J, Peters BA, Jacobs EJ, Gapstur SM, et al. Human oral microbiome and prospective risk for pancreatic cancer: a population-based nested case-control study. Gut. 2016.

11. Peters BA, Wu J, Pei Z, Yang L, Purdue MP, Freedman ND, et al. Oral microbiome composition reflects prospective risk for esophageal cancers. Cancer Res. 2017.

12. Takahashi N. Oral Microbiome Metabolism: From "Who Are They?" to "What Are They Doing?". J Dent Res. 2015;94(12):1628-37.

13. Wu GD, Chen J, Hoffmann C, Bittinger K, Chen YY, Keilbaugh SA, et al. Linking long-term dietary patterns with gut microbial enterotypes. Science. 2011;334(6052):105-8.

14. Wu GD, Chen J, Hoffmann C, Bittinger K, Chen YY, Keilbaugh SA, et al. Gut microbiota composition correlates with diet and health in the elderly. Nature. 2012;488(7410):178-84.

15. World Health Organization. Guideline: Sugars Intake for Adults and Children. Geneva (CHE): WHO; 2015.

16. He B, Long W, Li X, Yang W, Chen Y, Zhu Y, et al. Sugar-Sweetened Beverages Consumption Positively Associated with the Risks of Obesity and Hypertriglyceridemia Among Children Aged 7-18 Years in South China. J Atheroscler Thromb. 2018;25(1):81-9.

17. Lei L, Rangan A, Flood VM, Louie JC. Dietary intake and food sources of added sugar in the Australian population. Br J Nutr. 2016;115:868-77.

18. Rangan AM, Randall D, Hector DJ, Gill TP, Webb KL. Consumption of 'extra' foods by Australian children: Types, quantities and contribution to energy and nutrient intakes. Eur J Clin Nutr. 2007;62:356-64.

19. Bell AC, Kremer PJ, Magarey AM, Swinburn BA. Contribution of 'non-core' foods and beverages to the energy intake and weight status of Australian children. Eur J Clin Nutr. 2005;59:639-45.

20. Moynihan PJ, Kelly SA. Effect on caries of restricting sugars intake: Systematic review to inform WHO Guidelines. J Dent Res. 2014;93(1):8-18.

21. WHO/FAO. Diet. Nutrition and the Prevention of Chronic Diseases. Geneva (CHE): WHO. 2003.

22. Bradshaw DJ, Lynch RJ. Diet and the microbial aetiology of dental caries: new paradigms. Int Dent J. 2013;63(Suppl 2):64-72.

23. Moynihan P, Petersen PE. Diet, nutrition and the prevention of dental diseases. Public Health Nutr. 2004;7:201-26.

24. Rudney JD, Jagtap PD, Reilly CS, Chen R, Markowski TW, Higgins L, et al. Protein relative abundance patterns associated with sucrose-induced dysbiosis are conserved across taxonomically diverse oral microcosm biofilm models of dental caries. Microbiome. 2015;3:69.

25. Sheiham A, James WP. Diet and Dental Caries: The Pivotal Role of Free Sugars Reemphasized. J Dent Res. 2015;94:1341-7. 
26. Adler CJ, Dobney K, Weyrich LS, Kaidonis J, Walker AW, Haak W, et al. Sequencing ancient calcified dental plaque shows changes in oral microbiota with dietary shifts of the Neolithic and Industrial revolutions. Nat Genet. 2013;45:450-5.

27. Signoretto C, Burlacchini G, Bianchi F, Cavalleri G, Canepari P. Differences in microbiological composition of saliva and dental plaque in subjects with different drinking habits. New Microbiol. 2006;29(4):293-302.

28. Schueller K, Riva A, Pfeiffer S, Berry D, Somoza V. Members of the Oral Microbiota Are Associated with IL-8 Release by Gingival Epithelial Cells in Healthy Individuals. Front Microbiol. 2017;8:416.

29. Moon JH, Lee JH. Probing the diversity of healthy oral microbiome with bioinformatics approaches. BMB Rep. 2016;49:662-70.

30. McLean JS. Advancements toward a systems level understanding of the human oral microbiome. Front Cell Infect Microbiol. 2014;4:98.

31. Wang Q, Garrity GM, Tiedje JM, Cole JR. Naive Bayesian classifier for rapid assignment of rRNA sequences into the new bacterial taxonomy. Appl Environ Microbiol. 2007;73(16):5261-7.

32. Oksanen J, Blanchet FG, Kindt R, Legendre P, Minchin P, O'Hara RB, et al. Vegan: Community Ecology Package. R Package Version. 2.0-10. CRAN. 2013.

33. Knight R, Beiko RG, Huttenhower C. Predictive functional profiling of microbial communities using 16S rRNA marker gene sequences. Nat Biotechnol. 2013;31(9):814-21.

34. Marsh PD. Microbial ecology of dental plaque and its significance in health and disease. Adv Dent Res. 1994;8:263-71.

35. Marsh PD. Are dental diseases examples of ecological catastrophes? Microbiology. 2003;149:279-94.

36. Shaddox LM. Walker CB.Treating chronic periodontitis: current status, challenges, and future directions. Clin Cosmet Investig Dent. 2010;2:79-91.

37. Keller MK, Kressirer CA, Belstrøm D, Twetman S, Tanner ACR. Oral microbial profiles of individuals with different levels of sugar intake. J Oral Microbiol. 2017;9(1):1355207.

38. Tian J, Qin M, Ma W, Xia B, Xu H, Zhang Q, et al. Microbiome interaction with sugar plays an important role in relapse of childhood caries. Biochem Biophys Res Commun. 2015;468(1-2):294-9.

39. Hao W, Xu H, Chen X, Zhou Q, Zhang P, Chen F, et al. Changes in dental plaque microbial richness and oral behavioral habits during caries development in young Chinese children. Caries Res. 2015;49:116e123.

40. Jiang W, Zhang J, Chen $\mathrm{H}$. Pyrosequencing analysis of oral microbiota in children with severe early childhood dental caries. Curr Microbiol. 2013;67:537e542.

41. Ling Z, Kong J, Jia P, Wei C, Wang Y, Pan Z, et al. Analysis of oral microbiota in children with dental caries by PCR-DGGE and barcoded pyrosequencing. Microb Ecol. 2010;60:677e690.

42. Jiang W, Ling Z, Lin X, Chen Y, Zhang J, Yu J, et al. Pyrosequencing Analysis of Oral Microbiota Shifting in Various Caries States in Childhood. Microb Ecol. 2014;67:962e969.

43. Conrads G, De Soet JJ, Song L, Henne K, Sztajer H, Wagner-Döbler I, et al. Comparing the cariogenic species Streptococcus sobrinus and S. mutans on whole genome level. J Oral Microbiol. 2014;6:26189.

44. Tanner AC, Kent RL Jr, Holgerson PL, Hughes CV, Loo CY, Kanasi E, et al. Microbiota of severe early childhood caries before and after therapy. J Dent Res. 2011;90:1298-305.

45. Johansson I, Witkowska E, Kaveh B, Lif Holgerson P, Tanner AC. Microbiome in populations with a low and high prevalence of caries. J Dent Res. 2016;95:80-6. 
46. Fragkou S, Balasouli C, Tsuzukibashi O, Argyropoulou A, Menexes G, Kotsanos N. Streptococcus mutans, Streptococcus sobrinus and Candida albicans in oral samples from caries-free and caries-active children. Eur Arch Paediatr Dent. 2016;17:367-75.

47. Klinke T, Urban M, Luck C, Hannig C, Kuhn M, Krämer N. Changes in Candida spp., mutans streptococci and lactobacilli following treatment of early childhood caries: a 1-year follow-up. Caries Res. 2014;8:24e31.

48. Al-Ahmad A, Roth D, Wolkewitz M, Wiedmann-Al-Ahmad M, Follo M, Ratka-Krüger $\mathrm{P}$, et al. Change in diet and oral hygiene over an 8-week period: effects on oral health and oral biofilm. Clin Oral Investig. 2010;14(4):391-6.

49. Paju S, Pussinen PJ, Suominen-Taipale L, Hyvönen M, Knuuttila M, Könönen E. J Clin Microbiol. 2009;47:235-8.

50. Torlakovic L, Klepac-Ceraj V, Ogaard B, et al. Microbial community succession on developing lesions on human enamel. J Oral Microbiol. 2012;4:1-7.

\section{Figures}
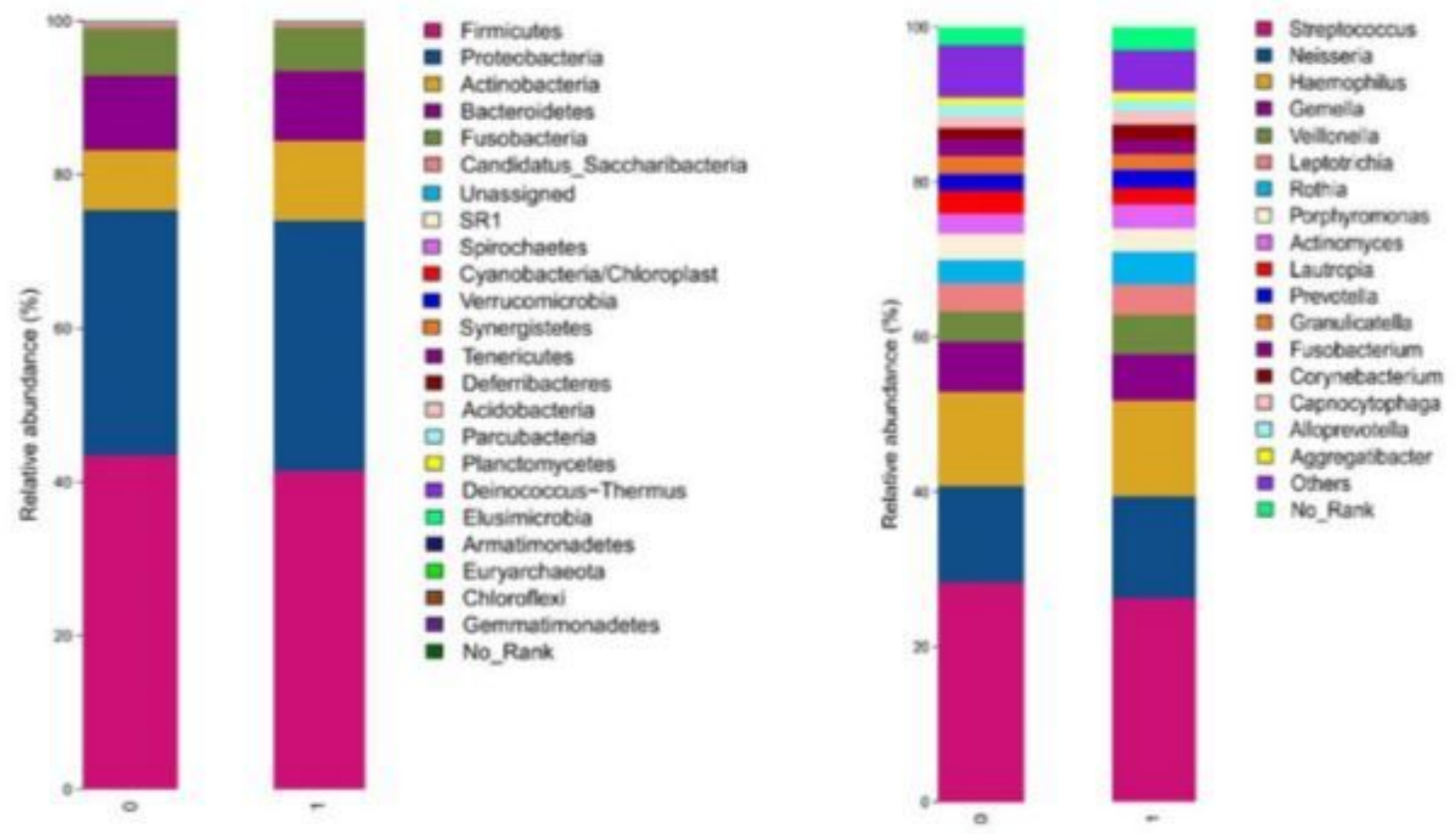

Figure 1

Relative abundance of oral microbiota at phylum (lift) and genus (right) level between groups with different SSBs intake ( 0 for $<1$ serving/week of SSBs intake, 1 for $\geq 1$ serving/week of SSBs intake) 

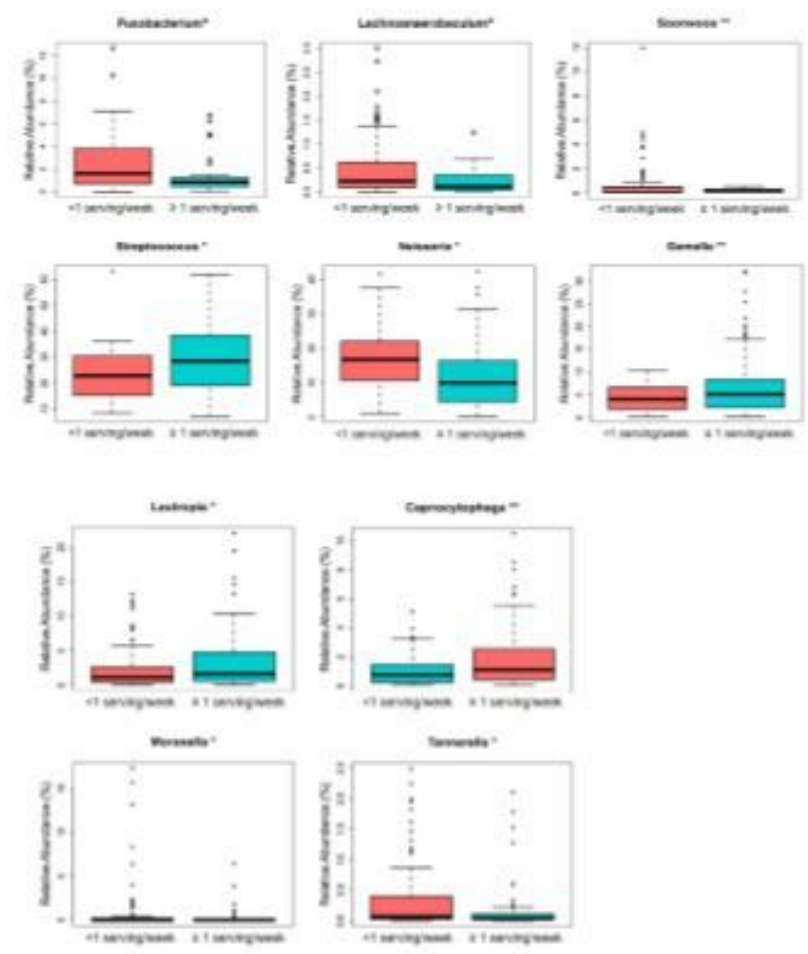

\section{Figure 2}

Metastats analysis of the difference of oral microbiota between different SSBs intake groups.

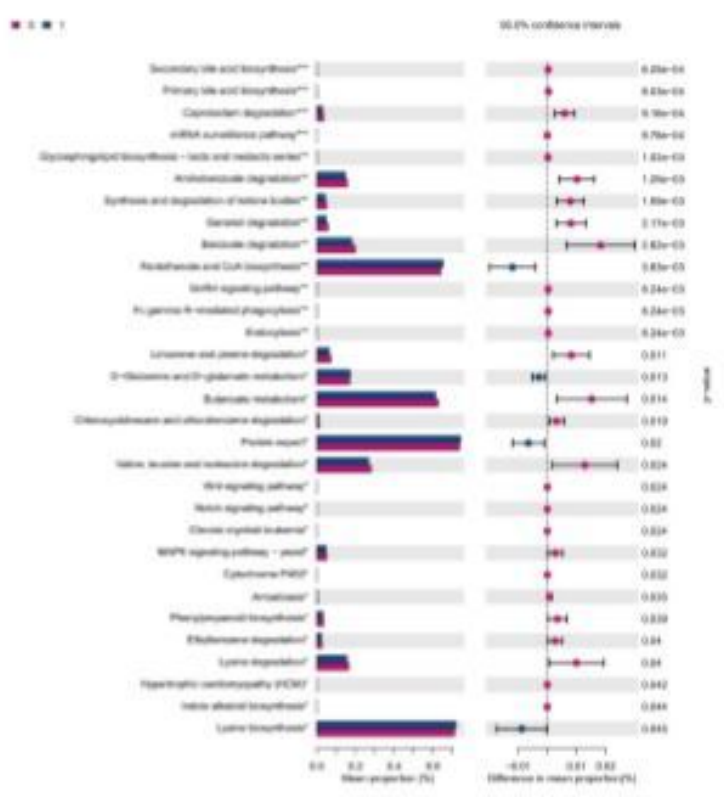

\section{Figure 3}

The KEGG analysis between groups with different SSBs intake(0 for $<1$ serving/week of SSBs intake, 1 for $\geq 1$ serving/week of SSBs intake).

\section{Supplementary Files}


This is a list of supplementary files associated with this preprint. Click to download.

- SupplementaryTable.docx 